\title{
Trauma abdominal em grávidas
}

\author{
Abdominal trauma in pregnant women
}

Gustavo Pereira Fraga ${ }^{1}$, Mario Mantovani², Ana Carla Mesquita ${ }^{3}$, Andrea Boldrin Soares ${ }^{3}$, Renato Passini Júnior ${ }^{4}$

\section{RESUMO}

Objetivos: avaliar os fatores indicativos (parâmetros clínicos e índices de gravidade fisiológicos e anatômicos) da evolução materna e fetal entre gestantes vítimas de trauma abdominal submetidas à laparotomia e discutir as particularidades do atendimento nesta situação. Métodos: análise retrospectiva dos prontuários de 245 mulheres com trauma abdominal e tratamento operatório, atendidas entre 1990 e 2002. Foram identificadas 13 gestantes com lesão abdominal submetidas à laparotomia. Para registro e análise estatística dos dados foram utilizados o protocolo Epi-Info 6.04 e o teste exato de Fisher, com intervalo de confiança de 95\%. Foram relacionados com a mortalidade fetal: escore na escala de coma de Glasgow, pressão arterial sistólica, índices de trauma (RTS, ATI, ISS) e lesão uterina. Resultados: a idade variou de 13 a 34 anos (média de 22,5). Seis mulheres $(46,2 \%)$ estavam no terceiro trimestre de gestação. O trauma penetrante correspondeu a 53,8\% das lesões e em seis dessas pacientes o mecanismo de trauma foi ferimento por projétil de arma de fogo. Três pacientes tiveram lesões uterinas, associadas com óbito fetal. Não houve óbito materno e a mortalidade fetal foi de 30,7\%. Não houve associação entre os índices de trauma e a mortalidade materna e fetal. A lesão uterina foi o único fator preditivo de risco para perda fetal $(\mathrm{p}=0,014)$. Conclusões: apesar da casuística pequena e de se tratar de estudo retrospectivo de gestantes com trauma grave, os achados deste estudo mostram que não há indicadores com boa acurácia para indicação da evolução materna e fetal.

PALAVRAS-CHAVE: Complicações na gravidez; Gravidez/complicações; Traumatismos abdominais; Violência doméstica; Mortalidade fetal

\section{ABSTRACT}

Purpose: to evaluate the predictors (clinical findings and physiological and anatomical scores) of the maternal and fetal outcomes among pregnant women victims of abdominal trauma who were submitted to laparotomy and to discuss particularities of assessment in this situation. Methods: retrospective analysis of the medical records of 245 women with abdominal trauma and surgical treatment, from 1990 to 2002. Thirteen pregnant women with abdominal injury were identified. All cases were registered in the Epi-Info 6.04 protocol and data were analyzed statistically by the Fisher exact test, with confidence interval of $95 \%$. Results: ages ranged from 13 to 34 years (mean of 22.5 ). Six women (46.2\%) were in the third trimester of pregnancy. Penetrating trauma accounted for $53.8 \%$ of injuries and in six of these patients the mechanism of trauma was gunshot wounds. Three patients had uterine injuries associated with fetal death. There were no maternal deaths and fetal mortality was $30.7 \%$. The use of trauma scores was not associated with maternal and fetal mortality. Uterine injury was the only predictive risk factor for fetal loss $(\mathrm{p}=0.014)$. Conclusions: this is a retrospective study analyzing a small number of pregnant women victims of severe trauma. However, the results show that there are no predictive accuracy scores to evaluate maternal and fetal outcomes.

KEYWORDS: Pregnancy complications; Pregnancy/complications; Abdominal injuries; Domestic violence; Fetal mortality

\section{Introdução}

As causas de morte maternas têm sofrido modificações na sua freqüência nas últimas décadas, demonstrando tendência de aumento das causas não obstétricas em relação às obstétricas, o que se

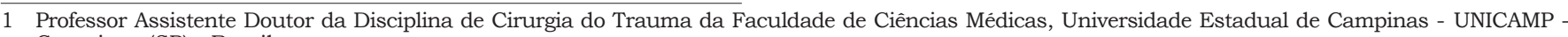
Campinas (SP) - Brasil.

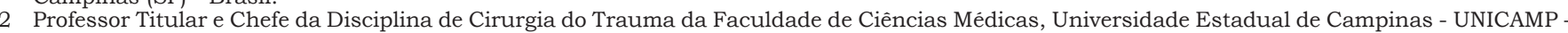
Campinas (SP) - Brasil.

3 Acadêmica de Medicina da Faculdade de Ciências Médicas, Universidade Estadual de Campinas - UNICAMP - Campinas (SP) - Brasil.

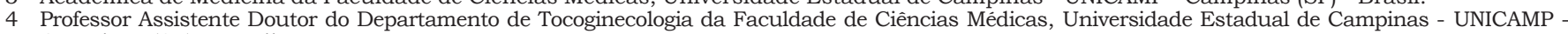
Campinas (SP) - Brasil.

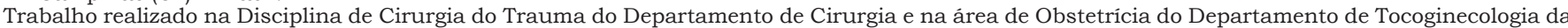
Faculdade de Ciências Médicas, Universidade Estadual de Campinas - UNICAMP - Campinas (SP) - Brasil.

Correspondência: Gustavo Pereira Fraga

Av. Coronel Silva Telles 211, apto. 03 - Cambui - 13024-000 - Campinas - SP - Telefone: (19) 9205-8167 - e-mail: fragagp@uol.com.br

Recebido em: 4/4/2005

Aceito com modificações em: 24/6/2005

Rev Bras Ginecol Obstet. 2005;27(9):541-7 
deve, em parte, às melhorias nos cuidados prénatais e na assistência ao parto, principalmente em paises mais desenvolvidos ${ }^{1}$. Atualmente, o trauma durante a gestação constitui uma das causas não obstétricas mais freqüentes de óbito materno $^{1-3}$. Traumas físicos ocorrem em aproximadamente 6 a $7 \%$ de todas as gestações, mas em menos de $1 \%$ das situações é necessária a hospitalização ${ }^{2-5}$. Esses números tendem a aumentar com a maior participação da mulher na população economicamente ativa e sua maior exposição às situações geradoras de trauma ${ }^{1,3}$.

As lesões por trauma ocorrem mais freqüentemente no segundo e terceiro trimestres de gravidez e são associadas a alto risco de perda fetal ${ }^{2}$. As alterações anatômicas e fisiológicas da gravidez podem alterar o padrão de uma lesão traumática e também interferir na avaliação e no tratamento da paciente ${ }^{1,2,6}$. Os princípios do atendimento à grávida vítima de trauma devem ser os mesmos de uma traumatizada não gestante, tendo como prioridade a reanimação materna ${ }^{1}$.

Considerando-se a incidência reduzida de hospitalização de gestantes por trauma e a pequena experiência diante dessa ocorrência, o objetivo do presente estudo é o de avaliar parâmetros clínicos, obstétricos e índices de gravidade fisiológicos e anatômicos a fim de determinar os fatores preditivos da evolução materna e fetal e discutir as particularidades do atendimento às gestantes vitimas de trauma abdominal, orientando obstetras e cirurgiões que atendem estas mulheres.

\section{Métodos}

A Disciplina de Cirurgia do Trauma é a responsável pelo atendimento às vítimas de trauma no Hospital das Clinicas (HC) da Unicamp, situado em Campinas, que tem população aproximada de 2,3 milhões de pessoas em sua região metropolitana.

$\mathrm{O}$ atendimento às gestantes vitimas de trauma segue os protocolos de rotina do serviço, baseados no Suporte Avançado de Vida no Trauma $\left(\mathrm{ATLS}^{\circledR}\right)^{7}$. São indicações de laparotomia: trauma abdominal com instabilidade hemodinâmica ou sinais de irritação peritoneal; trauma abdominal penetrante com violação de cavidade peritoneal; trauma contuso com métodos de imagem ou lavado peritoneal diagnóstico mostrando hemoperitônio (sem lesão de víscera maciça) ou sinais de lesão de víscera oca ${ }^{7}$. A avaliação obstétrica das pacientes é realizada em conjunto com a equipe do Departamento de Tocoginecologia do Centro de Atenção Integral à Saúde da Mulher (CAISM).
No período de janeiro de 1990 a dezembro de 2002, foram realizadas 1970 laparotomias em vítimas de trauma abdominal e registradas em protocolo Epi-Info 6.04. Nesta população houve predomínio do sexo masculino (1725 casos - $87,6 \%$ ) e o tipo de trauma mais freqüente foi o penetrante (1302 casos - 66\%). Analisando apenas o gênero feminino ( 245 casos - 12,4\%), observamos que 206 casos $(84,1 \%)$ encontravam-se na faixa entre $12 \mathrm{e}$ 43 anos, ou seja, na fase reprodutiva, sendo o trauma fechado o tipo mais freqüente (124 casos $50,6 \%)$. Entre as mulheres submetidas a laparotomia, a gravidez foi comprovada em 13 casos $(0,66 \%$ do total de laparotomias realizadas no periodo, correspondendo a 5,3\% das mulheres operadas e $6,3 \%$ das mulheres em idade reprodutiva submetidas à cirurgia), constituindo a casuística deste estudo retrospectivo.

Foi considerado critério de inclusão no estudo as grávidas com trauma abdominal submetidas à laparotomia. Foram excluídos casos de gestantes com traumatismos menores que ficaram apenas em observação na unidade de emergência, uma vez que a maioria destes casos não é encaminhada para serviço de referência.

Os seguintes dados foram considerados para a análise: idade da paciente; idade gestacional (dividida em $1^{\circ}, 2^{\circ}$ e $3^{\circ}$ trimestres); mecanismo do trauma; parâmetros fisiológicos na admissão hospitalar (escala de coma de Glasgow ${ }^{8}$, pressão arterial sistólica e freqüência respiratória); sinais clínicos e obstétricos; sinais de vitalidade fetal; métodos diagnósticos complementares; lesões abdominais e outras associadas; indices de trauma; tratamento e necessidade de hemocomponentes e evolução materno-fetal.

A todas as gestantes foram aplicadas escalas internacionais de medida de gravidade (indices de trauma): Escore de Trauma Revisado (Revised Trauma Score ou RTS), Índice de Gravidade da Lesão (Injury Severity Score ou ISS), TRISS (Probabilidade de Sobrevida) e o Índice de Trauma Abdominal (Abdominal Trauma Index ou ATI) ${ }^{9-13}$. O RTS é índice fisiológico com valor máximo de 7,84 (que representa paciente admitida em boas condições clínicas) e utiliza para o cálculo a escala de coma de Glasgow, a pressão arterial sistólica e a freqüência respiratória ${ }^{8,9}$.

O ISS é índice anatômico que em cada segmento corpóreo considera a lesão mais grave e seleciona os três segmentos que apresentaram lesão com maior pontuação, elevando-se cada um destes valores ao quadrado, com a soma dos mesmos resultando o ISS. O ISS varia de 0 a 75 , com pontuação de gravidade crescente; valores superiores a 25 representam traumatismos graves ${ }^{10}$. 
O TRISS é índice misto que é obtido após associar o RTS, o ISS, a idade da paciente (menor ou igual a 54 anos ou maior que 54 anos) e o tipo de traumatismo (fechado ou penetrante), sendo que o valor obtido representa a probabilidade de sobrevida estimada para a traumatizada ${ }^{11}$.

O ATI é outro índice anatômico no qual o escore de lesão de cada órgão intra-abdominal é o produto do fator de risco pela gravidade da lesão neste órgão, sendo que a soma dos escores de todos os órgãos lesados representa o ATI. Ao se calcular o ATI, pontuação superior a 25 é fator indicativo para ocorrência de complicações ${ }^{12}$.

As lesões uterinas foram classificadas de acordo com a escala de gravidade da Associação Americana de Cirurgia do Trauma ${ }^{14}$ (Quadro 1).

Quadro 1 - Escala para classificação de lesão uterina em gestantes

\begin{tabular}{|ll|}
\hline Grau & Lesão \\
\hline I & Contusão/hematoma (sem descolamento de placenta) \\
II & $\begin{array}{l}\text { Laceração superficial }(\leq 1 \mathrm{~cm}) \text { ou descolamento de placenta } \\
\text { parcial }<25 \%\end{array}$ \\
& Laceração profunda $(>1 \mathrm{~cm})$ ocorrendo no segundo trimestre \\
& ou descolamento de placenta $>25 \%$ e $<50 \%$ \\
IV & Laceração profunda $(>1 \mathrm{~cm})$ no terceiro trimestre \\
& Laceração envolvendo a artéria uterina \\
& $\begin{array}{l}\text { Laceração profunda }(>1 \mathrm{~cm}) \text { com }>50 \% \text { de descolamento de } \\
\text { placenta } \\
\text { R }\end{array}$ \\
& Rescolura uterina no segundo ou terceiro trimestre \\
&
\end{tabular}

Todos os casos foram registrados em protocolo Epi-Info 6.04. Conforme a natureza das variáveis estudadas, utilizou-se para análise dos resultados o teste exato de Fisher. O nível crítico para rejeição da hipótese da nulidade, a partir do qual a diferença foi considerada como estatisticamente significativa, foi de $5 \%(\mathrm{p} \leq 0,05)$, com intervalo de confiança de $95 \%$.

As seguintes variáveis foram relacionadas com a mortalidade fetal: escore na escala de coma de Glasgow (pontuação máxima igual a 15; ou alteração do nivel de consciência, escore inferior ou igual a 14), pressão arterial sistólica (PAS, estável hemodinamicamente, PAS superior ou igual a $90 \mathrm{mmHg}$; ou instável, PAS inferior a $90 \mathrm{mmHg}$ ), RTS (pontuação máxima igual a 7,84 ; ou alteração do estado fisiológico, inferior a 7,84), ATI (pontuação inferior ou igual a 25; ou superior a 25), ISS (pontuação inferior ou igual a 25; ou superior a 25) e lesão uterina (presente ou ausente).

A presente pesquisa foi previamente aprovada pelo Comitê de Ética em Pesquisa da Facul- dade de Ciências Médicas (FCM) da Universidade Estadual de Campinas (UNICAMP).

\section{Resultados}

Foram identificadas 13 gestantes vítimas de trauma e submetidas a laparotomia. A faixa etária variou de 13 a 34 anos, com média de 22,5 anos. Quanto à idade gestacional, três pacientes $(23,1 \%)$ estavam no primeiro trimestre, quatro $(30,7 \%)$ no segundo e outras seis $(46,2 \%)$ encontravam-se no terceiro trimestre de gravidez.

Houve predomínio dos traumas penetrantes (7 casos - 53,8\%), sendo o mecanismo mais freqüente o ferimento por projétil de arma de fogo (FPAF), conforme apresentado na Tabela 1. Entre os mecanismos de trauma contuso ( 6 casos - 46,2\%), predominaram os acidentes automobilísticos.

\begin{tabular}{lcc} 
Tabela 1 - Número de casos e porcentagem conforme o mecanismo de trauma \\
\hline Tipo de Trauma & \multicolumn{2}{c}{ Gestantes } \\
& Número & $\%$ \\
\hline Trauma penetrante & 7 & 53,8 \\
Arma branca & 1 & 7,7 \\
Arma de fogo & 6 & 46,2 \\
Trauma fechado & 6 & 46,2 \\
Atropelamento & 1 & 7,7 \\
Acidente automobilístico & 5 & 38,5 \\
Total & 13 & 100 \\
\hline
\end{tabular}

$\mathrm{Na}$ admissão hospitalar, duas pacientes $(15,4 \%)$ estavam inconscientes, com escore na escala de coma de Glasgow inferior ou igual a oito, e outras duas $(15,4 \%)$ foram admitidas com instabilidade hemodinâmica, com PAS inferior a 90 mmHg. O RTS variou de 5,38 a 7,84 , sendo que oito pacientes $(61,5 \%)$ obtiveram a pontuação máxima; a média foi de 7,25 e a mediana de 7,82 .

$\mathrm{O}$ exame obstétrico realizado na admissão mostrou que apenas uma paciente, com 36 semanas de gestação, vítima de acidente automobilístico, apresentava sangramento vaginal, sendo operada e identificada lesão esplênica e uterina grau I, tendo evoluído com óbito fetal.

Quanto ao batimento cardiaco fetal (BCF) na admissão, em nove gestantes foi considerado como presente pela ausculta com dopplerfluxometria ou avaliação com ultra-sonografia, e em duas destas pacientes $(22,2 \%)$ havia sinais de sofrimento fetal. Nestas, apesar do tratamento cirúrgico instituído, o concepto evoluiu para óbito. Em duas pacien- 
tes no primeiro trimestre de gravidez, não foi pesquisado $\mathrm{BCF}$ antes da cirurgia, e uma delas, com RTS de 5,67, evoluiu com abortamento. Outra grávida, com idade gestacional de 24 semanas, em que não foi pesquisado o $\mathrm{BCF}$, chegou em choque hipovolêmico, evoluindo com parada cardiorrespiratória e toracotomia de reanimação na chegada ao centro cirúrgico. Mãe e feto sobreviveram. Resultado contrário foi obtido em feto de gestante de 30 semanas vitima de FPAF em abdome, com lesão uterina grau $\mathrm{V}$, já que na admissão havia sido detectado óbito fetal.

Métodos propedêuticos complementares para pesquisa de lesão abdominal foram indicados em quatro pacientes $(30,8 \%)$. Estas eram vítimas de trauma contuso e foram avaliadas por meio de ultra-sonografia de abdome, que evidenciou líquido livre na cavidade em todos estes casos, além de diagnosticar lesão uterina em uma paciente. Em uma delas foi associada lavagem peritoneal diagnóstica, que se mostrou positiva para hemoperitônio.

As 13 gestantes foram tratadas por laparotomia, com intervalo de tempo entre a admissão e a cirurgia de no máximo duas horas. A Tabela 2 cita os órgãos e vísceras que foram lesados. Em duas pacientes $(15,4 \%)$ vítimas de FPAF a laparotomia foi considerada não terapêutica. Três grávidas $(23,1 \%)$ no terceiro trimestre de gestação tiveram lesão uterina, duas delas apresentaram lesão grau V (uma por FPAF e outra por trauma fechado, em que foi indicado histerectomia) e outra, grau I (por trauma fechado).

Tabela 2 - Número de casos e porcentagem de lesão em órgãos específicos em gestantes vítimas de trauma, conforme as lesões identificadas na laparotomia.

\begin{tabular}{lcc}
\hline & \multicolumn{2}{c}{ Gestantes } \\
Órgão afetado & Número & $\%$ \\
\hline Baço & 5 & 38,5 \\
Fígado & 5 & 38,5 \\
Útero & 3 & 23,1 \\
Rim & 3 & 23,1 \\
Cólon/reto & 2 & 15,4 \\
Jejuno/íleo & 2 & 15,4 \\
Estômago & 1 & 7,7 \\
Pâncreas & 1 & 7,7 \\
Sem lesão & 2 & 15,4 \\
\hline
\end{tabular}

O ATI variou de 1 a 39, com média de 13,5 e mediana de 13. Apenas duas pacientes $(15,4 \%)$ tinham ATI superior a 25. Lesões em outros segmentos corpóreos foram identificadas em cinco pacientes $(38,5 \%)$. A média do ISS foi de 23,9 e a mediana de 19. Quatro pacientes (30,8\%) apresentavam ISS maior que 25 .

Seis pacientes $(46,2 \%)$ tiveram o parto desencadeado pelo trauma, quatro delas $(66,7 \%) \mathrm{com}$ óbito fetal. Em cinco $(83,3 \%)$ foi indicada cesárea e uma paciente $(16,7 \%)$ evoluiu com aborto.

Não houve óbito materno nesta casuística e a mortalidade fetal foi de 30,7\% (quatro casos, um no primeiro trimestre e três no terceiro trimestre de gestação). A Tabela 3 apresenta análise de fatores preditivos para complicações relacionadas com a mortalidade fetal, pela qual se observa que a lesão uterina foi a única variável associada a um pior prognóstico.

Tabela 3 - Análise de fatores preditivos para complicações relacionadas com a mortalidade fetal em gestantes vítimas de trauma.

\begin{tabular}{|c|c|c|c|}
\hline \multirow[b]{2}{*}{ Fatores } & \multicolumn{2}{|c|}{ Mortalidade fetal } & \multirow[t]{2}{*}{$\mathrm{p}$} \\
\hline & Número & $\%$ & \\
\hline \multicolumn{4}{|l|}{ Glasgow } \\
\hline$=15$ & $1 / 8$ & 12,5 & 0,118 (NS) \\
\hline$\leq 14$ & $3 / 5$ & 60,0 & \\
\hline \multicolumn{4}{|c|}{ Pressão arterial sistólica } \\
\hline$\geq 90 \mathrm{mmHg}$ & $4 / 11$ & 36,4 & 0,461 (NS) \\
\hline$<90 \mathrm{mmHg}$ & - & - & \\
\hline \multicolumn{4}{|c|}{ Escore de Trauma Revisado (RTS) ${ }^{9}$} \\
\hline$=7,84$ & $2 / 8$ & 25,0 & 0,510 (NS) \\
\hline$<7,84$ & $2 / 5$ & 40,0 & \\
\hline \multicolumn{4}{|c|}{ Índice de Trauma Abdominal $(\mathrm{ATI})^{12}$} \\
\hline$\leq 25$ & $3 / 11$ & 27,3 & $0,538(\mathrm{NS})$ \\
\hline$>25$ & $1 / 2$ & 50,0 & \\
\hline \multicolumn{4}{|c|}{ Lesão uterina } \\
\hline presente & $3 / 3$ & 100,0 & 0,014 \\
\hline ausente & $1 / 10$ & 10,0 & \\
\hline \multicolumn{4}{|c|}{ Índice de Gravidade da Lesão (ISS) ${ }^{10}$} \\
\hline$\leq 25$ & $2 / 9$ & 22,2 & 0,358 (NS) \\
\hline$>25$ & $2 / 4$ & 50,0 & \\
\hline
\end{tabular}

Apenas uma paciente tinha TRISS menor que 0,50 , e esta sobreviveu, assim como seu concepto. A média do TRISS foi de 0.90 e a mediana de 0,99 . Nove gestantes $(69,2 \%)$ apresentaram TRISS de 0,99. A morbidade materna foi de 15,4\% com duas gestantes apresentando complicações pulmonares, que evoluíram satisfatoriamente com tratamento clínico. 


\section{Discussão}

Trauma durante a gestação é uma das causas não obstétricas mais freqüentes de morbimortalidade materna ${ }^{1-3,15}$. Isso não é surpreendente, uma vez que as vítimas de trauma encontram-se em sua maior parte na faixa etária correspondente à idade reprodutiva e o trauma representa a principal causa de morte na população abaixo dos 44 anos de idade ${ }^{7,16}$.

Nas últimas décadas, a freqüência das causas de morte materna tem sofrido mudanças. As causas obstétricas de óbito materno, as mais freqüentes no país, vêm diminuindo, apesar de ainda altas, em grande parte devido às melhorias nos cuidados pré-natais, na assistência ao parto e à conseqüente redução progressiva da mortalidade por hipertensão, hemorragia e infecção. Concomitantemente, as causas não obstétricas vêm ganhando importância, provavelmente pela crescente participação da mulher na população economicamente ativa e sua maior exposição às situações de trauma, chegando a representar 46,3\% das mortes de mulheres grávidas em um estudo realizado em Illinois, EUA, em 1992 ${ }^{1-3,17}$.

A literatura aponta que a média de idade das gestantes acometidas por trauma varia em torno de 23 a 26 anos, atingindo mulheres na faixa etária de 14 a 42 anos e ocorre mais freqüentemente no segundo e terceiro trimestres de gestação ${ }^{1-3,17}$. Os dados obtidos nesta casuística corroboram esta informação: a média de idade das pacientes foi de 22,5 anos e houve predomínio do trauma no segundo e terceiro trimestres de gravidez.

Os traumatismos fechados são descritos como os responsáveis pela grande maioria dos traumas na gestação ${ }^{1-4,18}$. Na maioria dos estudos predomina o acidente automobilístico como o mecanismo de trauma mais comum, o que poderia estar relacionado com o aumento do número de mulheres motoristas e a maior autonomia das gestantes para atividades cotidianas ${ }^{2,16}$.

Diferentemente do encontrado na literatura, nesta casuística o trauma penetrante foi a principal causa de trauma em gestantes $(53,8 \%)$, predominando as lesões por projétil de arma de fogo, o que pode estar relacionado aos altos índices de violência na cidade e região.

O trauma fechado correspondeu, no presente trabalho, a $46,2 \%$ dos casos, com predominio dos acidentes automobilísticos (38,5\%). Nos casos analisados não foram observados espancamento e quedas. Embora sejam freqüentes na gravidez, a maioria das quedas representam traumas leves que não requerem hospitalização, o que pode explicar sua ausência nessa casuística. No caso dos espancamentos, embora apareçam na literatura como importante causa de trauma em gestantes, nem sempre acarretam necessidade de laparotomia e, possivelmente, são subnotificados, já que a maioria das agressões são realizadas pelo marido ou companheiro ${ }^{1,2,16}$.

As prioridades do atendimento inicial adotadas para uma gestante vitima de traumatismo fisico são as mesmas aplicadas àquela não grávida ${ }^{7}$. O melhor tratamento inicial para o feto, além de sua avaliação precoce, é a adoção das medidas de reanimação para a mãe, uma vez que a mortalidade materna é uma das causas mais comuns de perda fetal e raramente ocorre dano fetal sem comprometimento materno, exceto nos casos de descolamento prematuro de placen$\mathrm{ta}^{2,3,19}$.

Para adequado atendimento à grávida traumatizada é necessário considerar as alterações anatômicas e fisiológicas da gravidez, mais evidentes no terceiro trimestre, tais como pressão arterial, freqüência cardíaca, volume sangüineo, posição do útero, bexiga, coração, etc. Modificações fisiológicas da gravidez interferem com a avaliação do estado hemodinâmico e podem provocar demora no estabelecimento de medidas corretivas ou de diagnóstico. O volume eritrocitário aumenta em torno de $40 \%$ na gravidez, o que permite maior perda volêmica sem expressão clínica. A pressão arterial está reduzida em 15 a 20\% e o débito cardíaco aumenta em até $50 \%{ }^{20}$. Essas alterações podem modificar o padrão de uma lesão traumática e também interferir na avaliação e no tratamento da paciente ${ }^{2,6,7}$.

Os órgãos mais freqüentemente lesados neste estudo foram baço $(38,5 \%)$, figado $(38,5 \%)$, útero $(23,1 \%)$ e rim $(23,1 \%)$. O intestino delgado foi afetado em apenas $15,4 \%$, o que é concordante com a literatura ${ }^{2,6,7}$

O aumento do útero na gestação compartimentaliza a cavidade peritoneal, protegendo muitas das vísceras abdominais; as alças intestinais são deslocadas para cima e lateralmente, reduzindo a chance de lesão intestinal em diversos traumatismos, principalmente em traumas abdominais contusos. Entretanto, traumas penetrantes que acometem o abdome superior nas fases tardias da gestação podem resultar em lesões intestinais complexas em decorrência deste deslocamento em direção cefálica. Concomitantemente, o aumento do útero o torna mais vulnerável ao trauma fechado ou penetrante ${ }^{6,7}$. Além disso, por estar relacionada anatomicamente ao útero, a bexiga torna-se mais suscetivel às lesões, 
embora isto não tenha sido observado na presente casuística ${ }^{6}$.

No atendimento a gestantes com mais de 26 semanas de gravidez vitimas de trauma, sempre que possivel deverá ser evitado que a paciente fique longos períodos em decúbito dorsal horizontal, pelo risco de compressão da veia cava inferior pelo útero gravídico. Esta recomendação se torna mais importante se houver necessidade de algum tipo de cirurgia, que pode demandar muitas horas com a paciente nesta posição ${ }^{21}$.

Embora a gravidez não aumente a morbimortalidade materna após o trauma, o prognóstico para o feto se agrava ${ }^{4,18,22}$. Trauma durante a gravidez está associado a aumento de risco de aborto, amniorrexe prematura, parto prematuro, descolamento prematuro de placenta, ruptura uterina e hemorragia feto-materna, relacionando-se com altas taxas de perda fetal. Mesmo lesões aparentemente insignificantes e que não envolvem o abdome podem resultar em mortalidade fetal significativa ${ }^{5,18,23}$.

Nesta casuística não houve óbito materno. Segundo vários autores, a mortalidade em gestantes traumatizadas é semelhante à de vítimas de trauma não gestantes ${ }^{1-4,17}$. A maior parte dos óbitos maternos se deve a choque hipovolêmico e a traumas craniencefálicos ${ }^{2,4,22}$. Vale ressaltar que apenas duas gestantes (15,4\%) foram admitidas hemodinamicamente instáveis e outras duas estavam em coma. Entretanto, na literatura, há outros fatores de risco específicos para mortalidade materna, entre eles lesão hepática e esplênica, hemorragia retroperitoneal (podendo estar associada à fratura pélvica), embolia por líquido amniótico e coagulação intravascular disseminada (CIVD) $)^{1,2,17}$.

As causas mais comuns para o óbito fetal nestas gestantes são a morte materna e o descolamento prematuro de placenta ${ }^{1,3,17}$. A mortalidade fetal observada neste estudo $(30,7 \%)$ está de acordo com as taxas citadas na literatura (1 a 34\%), destacando-se o fato de estas pacientes serem de maior risco, uma vez que os traumas foram graves e necessitaram de laparotomia ${ }^{1,3,4,18}$.

Dos quatro óbitos encontrados, um ocorreu no primeiro trimestre de gestação e três no terceiro. O predomínio de óbito fetal no último trimestre de gravidez encontrado nesta casuística também foi descrito em vários outros estudos e, provavelmente, está associado à maior incidência de trauma e maior gravidade que o trauma abdominal acarreta ao final da gestação ${ }^{2,3}$.

As lesões fetais secundárias aos traumas maternos são, mais freqüentemente, indiretas e, em sua maioria, conseqüentes à hipovolemia materna e hipoperfusão placentária. As diretas, menos comuns, são usualmente traumatismos cranianos associados a fraturas pélvicas, predominantes no terceiro trimestre, quando a cabeça do feto já está insinuada. Situações de desaceleração súbita em acidentes automobilísticos podem acarretar graves lesões fetais ${ }^{2}$.

A ruptura uterina é lesão específica da gestação, mas é rara. Quando ocorre, a mortalidade fetal chega quase a $100 \%^{1,2,22}$. O trauma sobre o útero pode levar a contrações uterinas e à ruptura de membranas ovulares. Quando ocorre lesão uterina com feto viável deve ser indicada cesárea, como observado no presente estudo ${ }^{1}$. Nos traumas penetrantes, se o útero for atingido, as lesões no feto podem ocorrer em 59 a $89 \%$ dos casos e as taxas de mortalidade fetal são de 41 a $71 \%^{6}$. No presente trabalho, em todos os casos em que houve lesão uterina ocorreu óbito fetal.

Muitos estudos têm citado como fatores de risco para perda fetal: morte materna, valores elevados de ISS, trauma abdominal, choque hemorrágico, sangramento vaginal e descolamento prematuro de placenta ${ }^{1,3,4,17,22}$.

Quanto à análise do prognóstico materno, a literatura mostra que pode ser feita a partir de indices de trauma como RTS, ISS e TRISS, indices rotineiramente adotados no atendimento aos pacientes traumatizados ${ }^{15,19}$. O ISS é índice utilizado para determinar a gravidade da lesão e estimar morbimortalidade após o trauma em população não gestante. Entretanto, o seu uso como fator indicativo de morbimortalidade materno-fetal ainda é controverso e tem sido discutido em muitos estudos ${ }^{5,15,16,23,24}$. Em relação ao índice que estima a probabilidade de sobrevida (TRISS) existem muitas criticas, especialmente em populações em que há predomínio de trauma penetrante ${ }^{13,15}$. Em estudo realizado no HC da Unicamp com 1380 pacientes submetidos a laparotomia por trauma abdominal, foram observados sensibilidade de $98,5 \%$, especificidade de $37,9 \%$ e erro de classificação de $11,7 \%$ ao se aplicar o TRISS ${ }^{13}$. Assim como descrito em outros estudos, os índices de trauma obtidos na presente casuística não mostraram associação com a mortalidade materna e nem fetal ${ }^{1-}$ $3,5,15,16$

Este é um estudo retrospectivo a partir de análise de prontuários de gestantes vítimas de trauma grave e submetidas à laparotomia e não pode ser generalizado para gestantes vítimas de traumas menores sem necessidade de hospitalização e intervenção cirúrgica. Além disso, a precisão dos resultados é limitada pelo pequeno tamanho da casuística. 
Os achados deste estudo permitem concluir que não há indicadores com boa acurácia para avaliação da evolução materna e fetal. Os índices de trauma não foram fatores preditivos de prognóstico para mãe ou feto, ao contrário da presença de lesão uterina, que foi o único fator associado a maior mortalidade fetal. Estudos futuros devem incorporar ferramentas de avaliação obstétrica e do trauma para desenvolver sistema de escore de estimativa de lesão que possa ser usado prospectivamente na avaliação prognóstica materna e fetal.

\section{Referências}

1. Corsi PR, Rasslan S, Oliveira LB, Kronfly FS, Marinho VP. Trauma na gestante: análise da mortalidade materna e fetal. Rev Col Bras Cir. 1998;26(2):79-83.

2. Spencer Netto FAC, Amorim MMR, Santos LC. Trauma e gravidez: parte I: artigo de revisão. Femina. 1999;27(1):11-8.

3. Shah KH, Simons RK, Holbrook T, Fortlage D, Winchell RJ, Hoyt DB. Trauma in pregnancy: maternal and fetal outcomes. J Trauma. 1998;45(1):83-6.

4. Esposito TJ, Gens DR, Smith LG, Scorpio R, Buchman T. Trauma during pregnancy: a review of 79 cases. Arch Surg. 1991;126(9):1073-8.

5. El-Kady D, Gilbert WM, Anderson J, Danielsen B, Towner D, Smith LH. Trauma during pregnancy: an analysis of maternal and fetal outcomes in a large population. Am J Obstet Gynecol. 2004;190(6):16618.

6. Kuhlmann RS, Cruikshank DP. Maternal trauma during pregnancy. Clin Obstet Gynecol. 1994;37(2):274-93.

7. American College of Surgeons. Advanced trauma life support instructor manual. Chicago: American College of Surgeons; 1997. p. 315-23.

8. Teasdale G, Jennett B. Assessment of coma and impaired consciousness. A practical scale. Lancet. 1974;2(7872):81-4.

9. Champion HR, Sacco WJ, Copes WS, Gann DS, Gennarelli TA, Flanagan ME. A revision of the trauma score. J Trauma. 1989;29(5):623-9.

10. Baker SP, O'Neill B, Haddon W Jr, Long WB. The injury severity score: a method for describing patients with multiple injuries and evaluating emergency care. J Trauma. 1974;14(3):187-96.
11. Boyd CR, Tolson MA, Copes WS. Evaluating trauma care: the TRISS method. Trauma Score and the Injury Severity Score. J Trauma. 1987;27(4):370-8.

12.Borlase BC, Moore EE, Moore FA. The abdominal trauma index: a critical reassessment and validation. J Trauma. 1990;30(11):1340-4.

13. Fraga GP, Mantovani M, Magna LA. Índices de trauma em pacientes submetidos à laparotomia. Rev Col Bras Cir. 2004;31(5):299-306.

14. Moore EE, Jurkovich GJ, Knudson MM, Cogbill TH, Malangoni MA, Champion HR, et al. Organ injury scaling. VI: extrahepatic biliary, esophagus, stomach, vulva, vagina uterus (nonpregnant), uterus (pregnant), fallopian tube, and ovary. J Trauma. 1995;39(6):1069-70.

15. Schiff MA, Holt VL. The injury severity score in pregnant trauma patients: predicting placental abruption and fetal death. J Trauma. 2002;53(5):9469.

16. Poole GV, Martin JN Jr, Perry KG Jr, Griswold JA, Lambert CJ, Rhodes RS. Trauma in pregnancy: the role of interpersonal violence. Am J Obstet Gynecol. 1996;174(6):1873-7.

17. Fildes J, Reed L, Jones N, Martin M, Barrett J. Trauma: the leading cause of maternal death. J Trauma. 1992;32(5):643-5.

18. Pearlman MD, Tintinalli JE. Evaluation and treatment of the gravida and fetus following trauma during pregnancy. Obstet Gynecol Clin North Am. 1991;18(2):371-81.

19. Rogers FB, Rozycki GS, Osler TM, Shackford SR, Jalbert J, Kirton O, et al. A multi- institutional study of factors associated with fetal death in injured pregnant patients. Arch Surg. 1999;134(11):1274-7.

20. Van Hook JW. Trauma in pregnancy. Clin Obstet Gynecol. 2002;45(2):414-24.

21.Lavery JP, Staten-McCormick M. Management of moderate to severe trauma in pregnancy. Obstet Gynecol Clin North Am. 1995;22(1):69-90.

22. Rothenberger D, Quattlebaum FW, Perry JF Jr, Zabel J, Fischer RP. Blunt maternal trauma: a review of 103 cases. J Trauma. 1978;18(3):173-9.

23. Ali J, Yeo A, Gana TJ, McLellan BA. Predictors of fetal mortality in pregnant trauma patients. J Trauma. 1997;42(5):782-5.

24. Theodorou DA, Velmahos GC, Souter I, Chan LS, Vassiliu P, Tatevossian R, et al. Fetal death after trauma in pregnancy. Am Surg. 2000;66(9):809-12. 